\title{
Mathematical models for forecast of geometrical parameters of assembly units
}

\author{
V.A. Pechenin ${ }^{1}$, M.A. Bolotov ${ }^{1}$, N.V. Ruzanov ${ }^{1}$

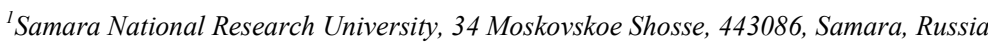

\begin{abstract}
The article describes two mathematical models for forecast of the geometric parameters of assembly units: the model of surface fitting and the combinatorial search model. The objects of modeling are a pair of mating cone rings. The first cone ring is characterized by the form deviation of the mating surface. The second cone is characterized by the runout of the outer and inner surfaces. Approval of the developed models was carried out through experimental studies of the assembly of two tailored and certified cone rings. A comparison of results of theoretical and experimental studies has shown that the developed models allow forecasting the geometric parameters of assembly units.
\end{abstract}

Kea words: mathematical model; form deviation; filtration; assembly; geometric parameter; coordinate measurements

\section{Introduction}

The performance characteristics of machines are in large part determined by the geometric precision of the parts and assembly units. The geometric precision of the assembly units is standardized by the assembly parameters and depends on the deviations of the form and location of the parts surfaces. The accuracy of the assembly parameters is achieved by the correct assignment of requirements for the deviations of the form and location of the parts surfaces and the choice of the process of assembly. A variety of sources $[1,2,3,4]$ is devoted to the technology of manufacturing and assembly of parts. Development of design of aviation and space equipment is constantly increasing the requirement for quality level of items. These requirements directly affect both the manufacturing processes of production and the technological processes of assembling individual parts and assembly units. Substantial reserves of increase of accuracy and productivity of assembly process are developed by using forecast and optimization models directly to manage the process of forming a required accuracy of assembly. The implementation of such reserves is possible when performing forecasting calculations based on real geometric models [5]. It is assumed that, the possible spatial positions of the parts achieved during mating through a plurality of surfaces can be calculated immediately before the assembly. In this case, the calculations must take into account the deviation of form and location of surfaces of the parts. Forecast assembly models are also useful for assembling micromechanical elements, for example microturbines [6, 7].

The paper describes two mathematical models that allow forecasting the relative positions of parts that have geometric deviations in the assembly. A description of the developed models is provided below.

\section{Object of research}

To research the forecast of assembly parameters, the pair of rings were manufactured from tool steel Cr12. Mating surfaces of rings are conical with specified deviations. The small ring opening is a precise cylindrical surface, the outer surface of the larger ring is the exact conical surface. This feature of the design is associated with the need to determine accurately the coordinates of the centers of cones during physical experiments and it is supported by the use of the basing procedure.

The internal conical ring has a form deviation, which can be described by the sum of the harmonic functions. The external ring has precise conical surfaces and the runout of the outer and inner surfaces. The parameters of mating surfaces of the rings are presented in Table 1.

Physical experiments were conducted on assembling conical rings as a check on the adequacy of the developed models for forecast of assembly parameters. The resulting positions were obtained by measuring the base surfaces of the conical rings in the assembled form by the coordinate measuring machine DEA Global Performance 07.07.07.

\begin{tabular}{lll} 
Table 1. Parameters of mating rings. & & \\
\hline Parameters & The inner ring & The outlet ring \\
\hline Height, mm & 36,295 & 39,971 \\
$\begin{array}{l}\text { An average radius of the circle of the lower } \\
\text { section, mm }\end{array}$ & 27,36 & 27,3487 \\
Form deviation, mm & 0,09 & \\
Runout of mating and base surfaces, $\mathrm{mm}$ & Absent & 0,389 \\
\hline
\end{tabular}

\section{Methods}

The following paragraph describes eveloped models for forecasting assembly states, as well as the procedure for generation of data on the geometry of the assembled rings using coordinate measurements. 


\subsection{Forecast of assembly parameters based on the model of fit of surfaces}

To calculate and forecast parameters of mating of parts consisting of surfaces with deviations, a model was developed; it is described in detail in [8] and that allows to calculate the mating of parts without taking into account deformations during the assembly process. The model uses an iterative algorithm to define the mating state, which involves iterative displacement of one interfacing surface relative to the other in the direction of the application vector $\overline{D_{1}}$ of the assembly force of the surfaces. In [8] the concept of a gap function $G(\bar{V})$ is introduced, it characterizes the achievement of the mating state of the surfaces of parts and depends on the vector of the geometric relationship of surfaces $\bar{V}$. To calculate the function $G(\bar{V})$, the best fit of the mating surfaces is performed at each stage, for this reason this approach is called the method of surfaces fitting (MSF). To perform the best fit procedure the iterative nearest-point algorithm (ICP) is used, it is presented in [9]. According to this algorithm at each iteration by the methods of non-linear optimization search, the rotation angles and components of the displacement vector are calculated along the coordinate axes. To avoid intersections of two surfaces, the system of inequalities is used, which are presented in [10], which imposes restrictions on the gap function.

\subsection{Combinatorial search model}

In the case of mating of a conical ring having the same form deviation along the generator line and an ideal conical surface, it is possible to find the assembly center in a simpler way rather than using the ICP approach. The essence of the approach is to find the parameters of a circle of minimal radius circumscribed around one of the sections of the external cone ring. To solve this problem, a combinatorial search model was developed basing on enumeration of combinations of $\mathrm{n}$ defining profile points by 3 points at a time without repetitions. Each set of 3 points defines a circle, and its center and radius are calculated. The parameters of the circles are found from the system of equations:

$$
\left\{\begin{array}{l}
\left(P_{x 1}-O_{x}\right)^{2}+\left(P_{y 1}-O_{y}\right)^{2}=R_{c}^{2}, \\
\left(P_{x 2}-O_{x}\right)^{2}+\left(P_{y 2}-O_{y}\right)^{2}=R_{c}^{2}, \\
\left(P_{x 3}-O_{x}\right)^{2}+\left(P_{y 3}-O_{y}\right)^{2}=R_{c}^{2} ;
\end{array}\right.
$$

where $P_{1}, P_{2}, P_{3}$ are points of the profile of the internal cone ring;

$O$ is the center of the circle passing through the points $P_{1}, P_{2}, P_{3}$;

$R_{c}$ is the radius of the circle passing through the points $P_{1}, P_{2}, P_{3}$.

To find the circles circumscribed around the search points, the distances from the center of the circle to all points of the profile are computed and compared with the radius of the circle $R_{c}$. If there are distances exceeding the radius $R_{c}$, then such combinations are not considered.

The circumscribed circles, which has the minimum radius $R_{c \text { min }}$, is chosen of all. It has a radius of the mating surface of the external ring, and the position of the center of the circle is the position of the center of the axis of the inner surface of the external ring. To find the displacement of the external ring along the z-axis (for example, focusing on the displacement of the upper end), it is necessary to calculate height of the defined section location. For this, the value of the applicator of the section of the internal ring is added to the value equal to:

$\Delta_{H}=\left(R_{c_{-} \text {min }}-R_{\text {upper_end }}\right) / \operatorname{tg}(\gamma / 2)$,

where $R_{\text {upper_end }}$ is the radius of the upper section of the external conical ring;

$\gamma$ is the angle at the vertex of the cone.

Thus, the position of the external ring along the $\mathrm{z}$ axis is calculated. It is possible to calculate the position of the centers during experimental studies graphically, taking into account the outrun of the surfaces of the external ring. The points of a circle with a diameter equal to the runout are calculated around the center of the mating surface of the external ring.

The more points of the surface of the internal ring are taken for the solution, the more accurate it will be, but the longer it will take to search through all the solutions. So, in the case of 120 setting points, the number of combinations which are to be considered equals $C_{360}^{3}=120 ! /((120-3) ! \cdot 3 !)=280840$. Accordingly, it is appropriate to use parallel computation and limit the number of search points used in the developed model.

\subsection{Simulation of models of real parts surfaces}

To calculate the assembly parameters using the models described above, information about the reference points and a mathematical description of the mating surfaces is necessary. Reverse engineering technology was used to obtain data on the surfaces of the conical rings under consideration, that involves measuring the surfaces by the coordinate measuring machine and further mathematical processing of the measurement data. The reverse engineering procedure applied to manufactured conical rings is presented in Figure 1.

During coordinate measurements of parts at the first stage their mathematical basing is made. The technology of mathematical basing of conical rings on CMM is based on measuring the surfaces by which you can specify the position of the coordinate axes of the part. Mathematical basing is a procedure consisting of calculation of the location of the part coordinate system (PCS) using the points of the base elements of the part previously measured in the machine coordinate system (MCS) to the subsequent 
transformation of the coordinates of the points of other component parts from the MCS to the PCS. Thus, mathematical basing means calculating the optimal transformation matrix $M$ which ensures the best fit of the measured points with the corresponding nominal points. The matrix contains three components of displacement along the coordinate axes $\Delta X, \Delta Y$ and $\Delta Z$, as well as three components of rotation around the coordinate axes $\Delta \Phi, \Delta \Theta$ and $\Delta \Psi$.

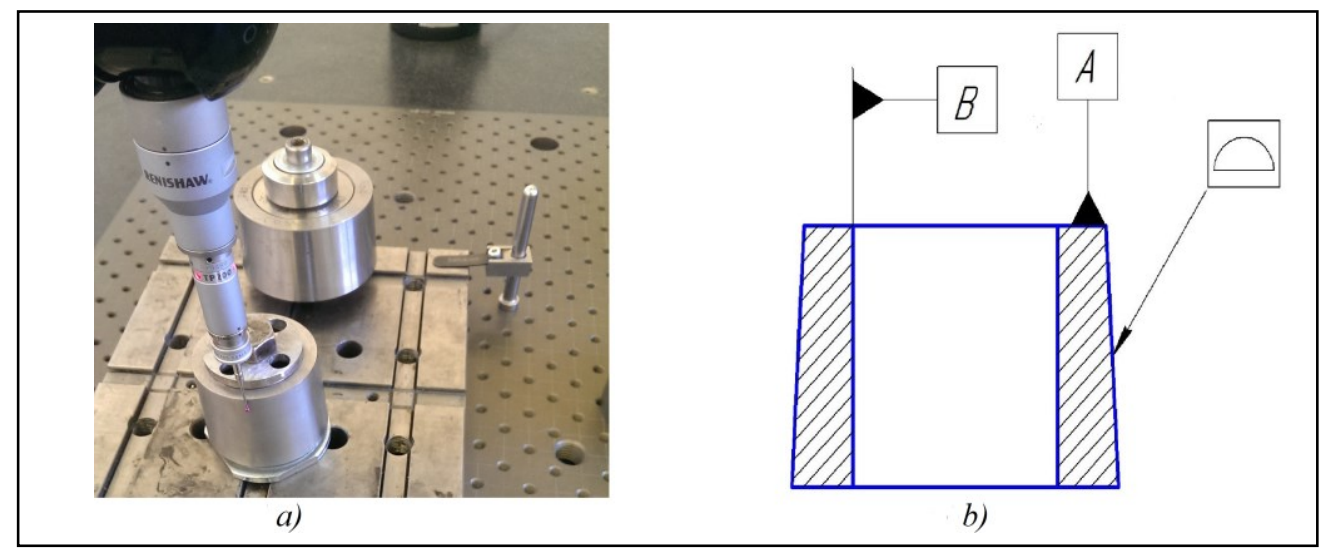

Fig. 1. Basing of the conical ring a) measurement by the CMM; b) scheme of conical ring basing.

Position of the main axis, the direction of the second axis and the center of the coordinate system are to be determined in the process of determination of the part coordinate system, the. For considering parts the direction of the rotation axis is defined by the butt end A (figure 1). The normal vector of the plane is collinear to the axis of the ring. The hole on the small ring and the outer surface of the external ring determine the centers of the axes. The hole points are measured in one section (base B). After measurement, the replacement element "circumference" is inscribed into the cloud of measured points. according to the method of least squares (LSM) The axis of the ring moves to the center of the circle. The cylinder axis is the axis of rotation. At the intersection of the end plane and the axis, the origin point is specified.

The basing procedure described above is carried out in two stages. At the first stage, the elements are measured manually by the smaller number of points (draft basing). At the second stage, more points are measured automatically that is clean basing.

Nevertheless, the coordinate system constructed on surfaces A and B may be out of the coordinate system of the mating surface, due to processing errors (non perpendicularity to the end, displacement of the center). To eliminate this error, the best comparison of the measured points of the mating surface with the mathematical model of the surface, along which coordinate system was processed and transformed, is performed. The mating surface is measured at list in three sections throughout the height and there are 300-360 points in each section.

The obtained parameters of matrix transformation $M$ are used in further experiments for basing of the part, i.e. it is not required to produce the best combination of surfaces each time.

The coordinates of the measured section points of the mating surface $P_{\text {meas }}$ are loaded into the MATLAB system.

The measured coordinates of the points contain various random components of errors caused by measurement errors, as well as by emissions from various factors atypically for the entire surface. For modern contact CMM such error is $0.5-4 \mu \mathrm{m}$ but when measuring complex curved surfaces or if condition is non-compliance with normal conditions this measurement error can be greater. During the processing of the measured data it is often necessary to remove the noise representing the inaccuracy of the measuring instrument $[11,12]$. The problems associated with the fall in the image processing occur $[13,14,15]$. There are an extensive research and reduction in the processing data, [16, 17]. It is necessary to filter such errors for more accurate measurement results and obtaining adequate estimates of the parameters of the measured rings. One of the most effective tools for filtering random errors is smoothing splines [18].

The smoothed spline $\hat{\varepsilon}$ of the set of deviations $\varepsilon$ minimizes the equation:

$p \sum_{i=1}^{n} w_{i}\left(\varepsilon\left(u_{i}\right)-\hat{\varepsilon}\left(u_{i}\right)\right)^{2}+(1-p) \int\left(D^{2} \hat{\varepsilon}(u)\right)^{2} d u \rightarrow \min$,

where $(u, \varepsilon)$ are approximated data (the point and magnitude of the deviation in it);

$p$ is the smoothing parameter $p \in[0,1]$;

$w$ is a vector of weights (it is taken as the vector of units).

Filtering with a parameter $p$ equal to 0.99 was used to filter the errors of the measured ring point arrays. To carry out the filtration, it is necessary to calculate the radius vectors of the measured points in each section. The radius vector of the i-th point is calculated by the equation:

$r_{P i_{-} \text {meas }}=\sqrt{P_{x i_{-} \text {meas }}^{2}+P_{y i_{-} \text {meas }}^{2}}$.

The deviation of the form at the points of the section $\delta_{F}$ is calculated as the difference between the radius vector of the point $r_{p}$ and the nominal radius of the cone in the cross section $r_{\text {cone }}$ :

$\delta_{F}=r_{p}-r_{\text {cone }}$. 
After filtration, the average deviation of the form at each point of each measured section is calculated. The values of calculated deviations at points were used to calculate the points of a new surface (taking into account manufacturing errors). In the MATLAB system the NURBS surface was automatically constructed and saved as the an *.igs file. The built surface was loaded in CAD system and the it was changed taking in account a new surface of the 3D model of the ring.

At the final stage, a remeasurement was carried out according to the procedure described above, in order to reduce the measurement errors that arise due to the discrepancy of the measured part to the reference model [19]. The measurements were re-processed according to foregoing sequence and the surface parameters were saved for modeling the assembly process.

\section{Results}

To obtain experimental data on the assembly parameters, 20 measurements of the rings in the assembled form were made by a coordinate measuring machine. The external ring in each measurement was rotated by an angle about the axis. To find the center of the inner mating surface of the external ring, a least-squares circle was inscribed in 20 measured points (Figure 2).

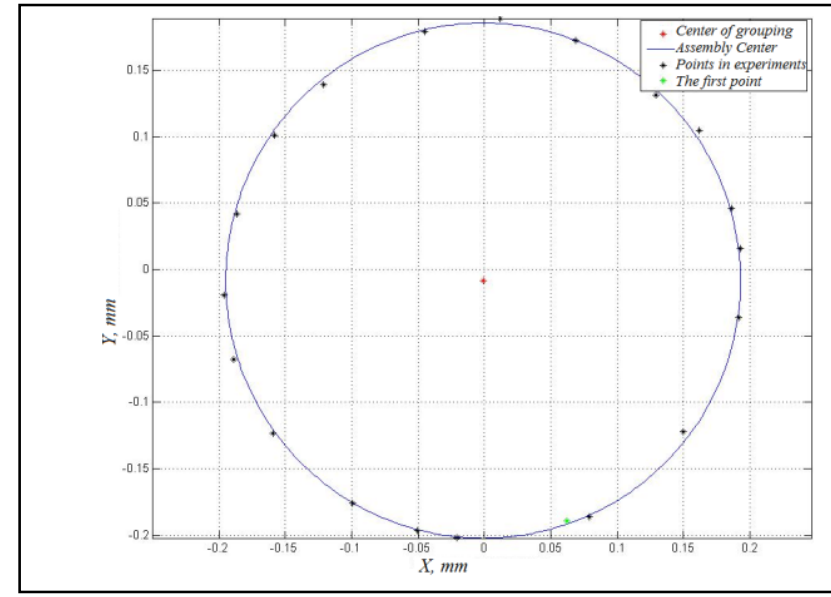

Fig. 2. A graphical solution for finding the center of mating and outrun.

The diameter of the circle characterizes the outrun of the external cone ring relative to the internal ring (Table 1). The nonparallelism of the axes of the mating cone rings results in additional error, which during the experiments was 0.005-0.014 mm.

Thus, the parameters characterizing the mating of the conical ring with the deviation of the form and the cone ring with the deviation of the location (outrun of the conical surfaces) are obtained.

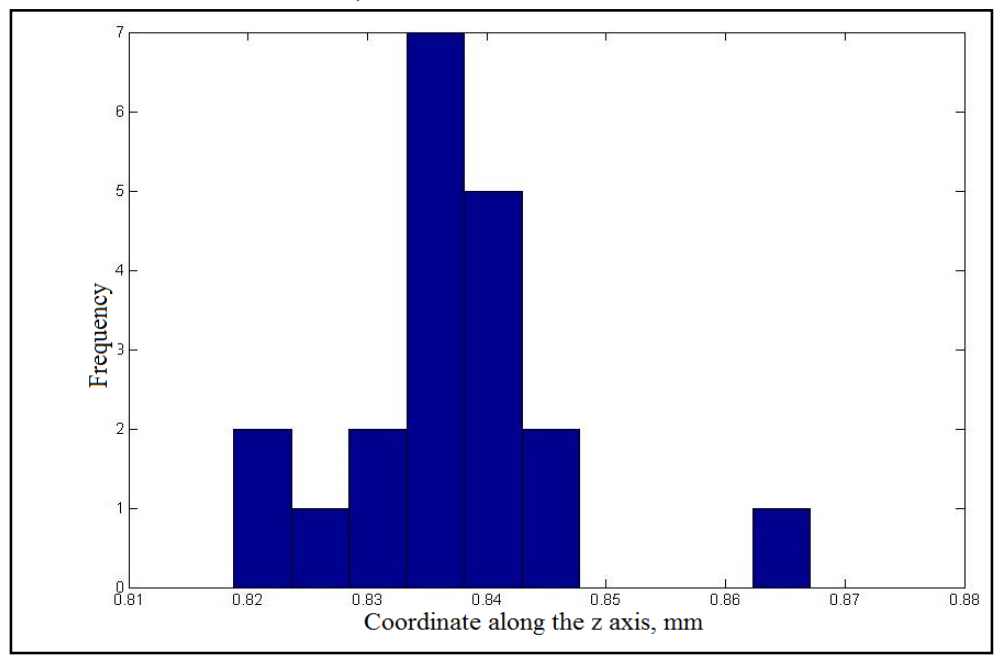

Fig. 3. Histogram of the coordinates along $z$.

At the same time, during assembly process various positions of the centers along the $\mathrm{z}$ axis were performed, that is explained by the action in the mating of rings of frictional forces, which are characterized by non-parallel axes during assembly process. Consequently, the action of friction forces leads to the appearance of an error relative to the calculated mating center. A histogram of the distribution of center values along the $\mathrm{z}$ axis is shown in Figure 3.

Further, due to fluctuations along the $\mathrm{z}$ axis during assembly, the calculated positions of the centers have difference from the ideal circle inscribed in them, which also means the possible magnitude of the errors in determination of the assembly center. Figure 4 shows histograms of the distances of oscillations of the radius vectors of points relative to the radius of an ideal circle determined by the method of least squares.

After experiments the ring assembly parameters were calculated using the MSF models and combinatorial search. To calculate the assembly parameters, the points of the surface of the internal ring calculated using reverse engineering procedures were used. The points of the complementary mating surface of the external ring were set as points of an ideal conical surface. 
A comparison of the resulting positions of the external conical ring in the assembly determined by MSF, the combinatorial search model and in physical experiments, is given in Table 2.

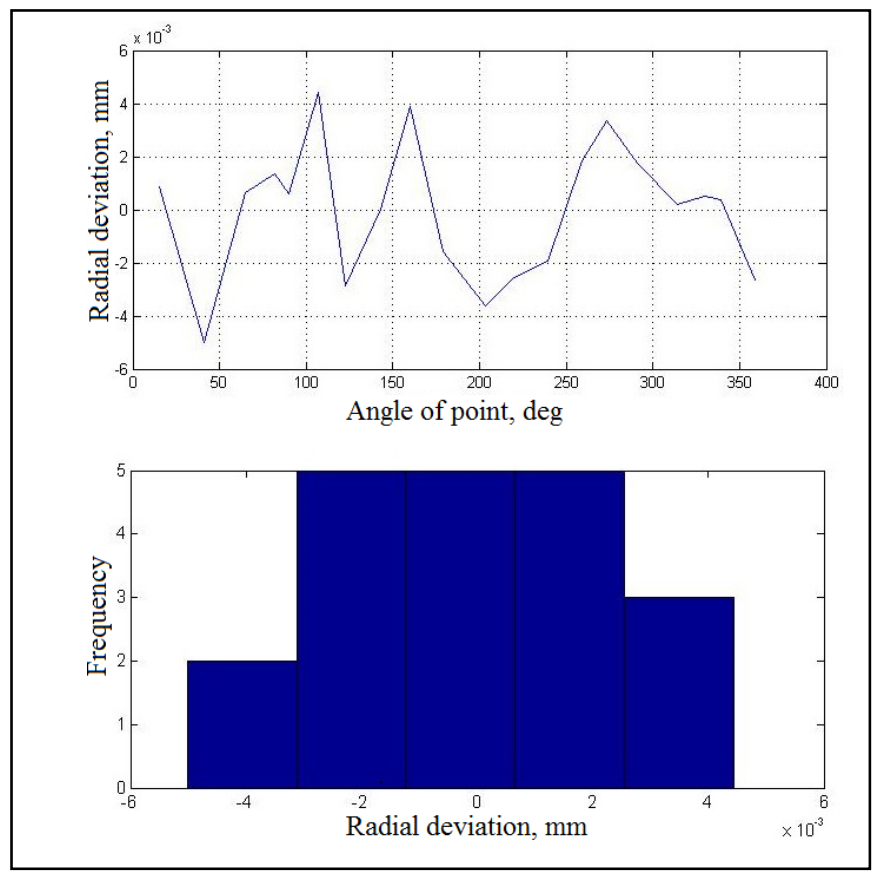

Fig. 4. Oscillations of the radius of the measured points of the centers.

Table 2. Comparison of the resulting coordinates of the position of the parts centers during assembly.

\begin{tabular}{llll}
\hline Coordinates of mating center & Experimental data & MSF & Combinatorial search model \\
\hline Along X, mm & $-0,0009$ & $-0,0018$ & $-0,0016$ \\
Along Y, mm & $-0,0083$ & $-0,0059$ & $-0,0069$ \\
Along Z, mm & $0,8188-0,8671$ & 0,9471 & 0,9353 \\
\hline
\end{tabular}

Comparing the results of the experimental solution and the solutions by simulation, it can be noted that both methods have rather high accuracy of the search for a solution along $X$ and $Y$ axes, and deviations don't exceed $2 \mu \mathrm{m}$ in the case of SMEs and $1 \mu \mathrm{m}$ using the combinatorial search model. Coordinates along the axis $Z$ have larger deviations, since deviations of micrometers in the plane $X O Y$ result in deviations of tens of micrometers along $Z$. The discrepancies are connected, first of all, with the fact that the frictional force of the surfaces was not taken into account in modeling and there is no misalignment of axes of cones during assembly. Deformations are also possible on contact of surfaces, but for such details as conical rings they are not significant.

\section{Conclusion}

The paper describes two mathematical models: model for combining surfaces and the combinatorial search model, that allow to forecast the geometric parameters of assembly units. The developed models are useful because they may forecast the relative positions of parts, which have geometric deviations. Comparison of simulation results with experimental data on the example of the assembly of conical rings showed fine precision in determination of the position of the center of the external ring axis. It is shown that the calculation of the errors of the assembly parameters can be made from the results of measurements of geometry by modern CMMs, which allow obtaining data on the points of surfaces in electronic form. The developed models can be used to forecast the assembly of critical parts of aviation equipment, such as shafts, discs, blades.

\section{Acknowledgement}

This work was supported by the Ministry of Education and Science of the Russian Federation in the framework of the implementation of the Program "Research and development on priority directions of scientific-technological complex of Russia for 2014-2020.

\section{References}

[1] Ermakov AI, Shklovets AO, Popov GM, Kolmakova DA. Investigation of the effect of the gas turbine compressor supports on gas flow circumferential nonuniformity. Research Journal of Applied Sciences 2014; 9(10):684-690.

[2] Popov GM, Goryachkin ES, Baturin OV, Kolmakova DA. Development of recommendations on building of the lightweight calculation mathematical models of the axial turbines of gas turbine engines. International Journal of Engineering and Technology 2014; 6(5): 2236-2243.

[3] Smelov VG, Sotov AV, Agapovichev AV. Research on the possibility of restoring blades while repairing gas turbine engines parts by selective laser melting. IOP Conference Series: Materials Science and Engineering 2016; 140: 012019. 
[4] Alexeev VP, Balyakin AV, Khaimovich AI. Influence of the direction of selective laser sintering on machinability of parts from 316L steel. IOP Conference Series: Materials Science and Engineering 2017;177(1): 012120.

[5] Kolmakova DA, Baturin OV, Popov GM. Effect of manufacturing tolerances on the turbine blades. ASME 2014 Gas Turbine India Conference, GTINDIA 2014; $1-10$.

[6] Morozov AA, Skidanov RV. Rotation of microturbine in complex vortex beams. Computer Optics 2013; 37(2): 203-207. (in Russian)

[7] Ganchevskaya SV, Skidanov RV. The microturbine rotation by not circular light beam formed by vortex Axicon . CEUR Workshop Proceedings 2016; 1638: 24-31.

[8] Bolotov MA, Pechenin VA, Murzin SP. Method for uncertainty evaluation of the spatial mating of high-precision optical and mechanical parts. Computer Optics 2016; 40(3): 360-369. DOI: 10.18287/2412-6179-2016-40-3-360-369.

[9] Besl PJ, Mckay ND. A method for registration of 3-D shapes. IEEE Transactions on Pattern Analysis and Machine Intelligence 1992; 14(2): 239-256.

[10] Pierce RS, Rosen D. Simulation of mating between nonanalytical programing formulation . Journal of Computing and Information Science in Engineering 2007; 7(4): 314-321.

[11] Soifer VA, Kupriyanov AV. Analysis and recognition of the nanoscale images: conventional approach and novel problem statement. Computer Optic 2011; 35(2):136-144.

[12] Myasnikov V, Popov SB, Sergeyev VV, Soifer VA. Computer Image Processing. Part I: Basic concepts and theory. VDM Verlag, 2009 ; 296 p.

[13] Kazanskiy NL, Khonina SN, Skidanov RV, Morozov AA, Kharitonov SI, Volotovskiy SG. Formation of images using multilevel diffractive lens. Computer Optics 2014; 38(3): 425-434.

[14] Borodin SA, Volkov AV, Kazanskiy NL. Device for analyzing nanoroughness and contamination on a substrate from the dynamic state of a liquid drop deposited on its surface. Journal of Optical Technology 2009; 76(7): 408-412.

[15] Kopenkov VN, Sergeev VV, Timbai EI. Regression restoration methods as applied to solve the problem of multidimensional indirect measurements. Pattern Recognition and Image Analysis 2011; 21(3): 501-504.

[16] Fleishman S, Drori I, Cohen-Or D. Bilateral mesh denoising. ACM Transactions on Graphics 2003; 22(3): 950-953.

[17] Abdul-Rahman HS, Scott PJ, Jiang XJ. Freeform surface filtering using the lifting wavelet. Precision Engineering 2013; 37(1): 187-202.

[18] De Boor C. A Practical Guide to Splines (Revised Edition). New York: Springer, 2001; 348 p.

[19] Mayer J, Mir Y, Trochu F, Vafaeesefat A, Balazinski M. Touch probe radius compensation for coordinate measurement using kriging interpolation. Proceedings of the Institution of Mechanical Engineers. Part B: Journal of Engineering Manufacture 1997; 211(1): 11-18. 